to attempt the destruction of pneumococci in the mouth secretion's, particularly among convalescents and those persons in intimate contact with cases of lobar pneumonia, in an effort to shorten the period of time in which convalescents or healthy carriers harbor pathogenic pneumococci in the mouth. Wadsworth ${ }^{5}$ has already tested the disinfecting power of saline solutions and bland alcoholic washes in ridding the mouth of pneumococci with somewhat encouraging results; more definitely pneumococcidal substances were not employed.

As the investigations of Morgenroth and Levy, Wright, ${ }^{7}$ Moore, ${ }^{8}$ and Cohen, Kolmer and Heist ${ }^{9}$ have shown the high pneumococcidal activity of ethylhydrocuprein hydrochlorid and, as found by the latter, ${ }^{9}$ that other cinchonics, as quinin and urea hydrochlorid and quinin bisulphate, also possess considerable pneumococcidal powers, we have made our studies on the disinfection of sputum and the mouth with solutions of these various cinchonics in a menstrutum of liquor thymolis, ${ }^{10}$ our experiments demonstrating that the latter solution alone in dilution of from $1: 4$ to $1: 10$ possesses some germicidal activity for pneumococci and aids in disguising the bitter taste of cinchona compounds. We have approached this problem mainly from the laboratory side, employing both normal mouth secretions harboring Type IV pneumococci and the sputum of pneumonia convalescents harboring Type I pneumococci, the pneumococcidal activity of the disinfectant under study being largely determined by mouse inoculation.

The results of our experiments with sputum have indicated that dilutions of ethylhydrocuprein hydrochlorid as high as $1: 30,000$ and even to $1: 160,000$ had appreciable and frequently well defined pneumococcidal activity, while a $1: 10,000$ dilution almost invariably disinfected sputum containing pneumococci, as determined by intraperitoneal injection in mice; with such cinchonics as quinin bistulphate, dilutions in sputum varying from $1: 10,000$ to $1: 20,000$ were found to possess well defined pneumococcidal activity.

After many trials, we found that $1: 10,000$ dilutions of ethylhydrocuprein hydrochlorid or quinin bisulphate, quinin hydrobromid, and other cinchonics in a $1: 10$ dilution of liquor thymolis, constitute mixtures that may be readily used by persons as mouth washes and gargles. The slightly bitter taste remaining after the use of any of these washes is readily removed by rinsing the mouth with plain water. Experiments consisting in mixing sputum containing Type I or Type IV pnetumococci with these solutions, and determining pneumococcidal activity by injecting portions into mice at varying intervals, have demonstrated pneumococcidal activity, particularly in the case of ethylhydrocuprein hydrochlorid.

In view of the established fact that a considerable percentage of persons in contact with cases of lobar pneumonia due to Types I and II pneumococci became carriers of these pneumococci, and that convalescents may carry them for varying intervals of time averag-

5. Wadsworth, A.: Jour. Infect. Dis., 1906, 3, 774

6. Morgenroth and Levy: Berl. kliz. Wchnschr., 1911, 48, 1561, 1650, 1779,1983

7. Wright, A.: Lancet, London, 1912, 2, 1633, 1701

8. Moore, H.: Jour. Exper. Med., 1915, 22, 269.

9. Cohen, S. S.; Kolmer, J. A., and Heist, G.? Jour. Infect. Dis., 1917, 20, 272 .

10. This solution, which is used in the Polyclinic Hospital as a substitute for liquor antisepticus, is prepared after the following formula: benzoic acid 64 grains; boric acid, 128 grains; thymol and menthol, each 16 grains; oil of eucalyptus, oil of wintergreen and oil monarda, each 4 drops; alcohol and glycerin, each 4 ounces; water sufficient for 16 ounces. ing from three to four weeks, the tise of a mouth wash and gargle of ethylhydrocuprein hydrochlorid is suggested as worthy of clinical trial by those persons who have come in contact with cases of lobar pneumonia, and also by the patients themselves and persons suffering with measles or other infections favoring the development of lobar pneumonia. The systematic use of 1: 10,000 solution of ethylhydrocuprein hydrochlorid in 1:10 dilution of liquor thymolis twice or more daily is not dangerous from the standpoint of toxicity due to swallowing portions of the drug, is not unpleasant, and may aid in ridding the mouth of virulent pneumococci, among contacts at least. Since this drug is scarce at present, one of the commoner cinchonics, as quinin bisulphate or quinin hydrobromid, may be substituted, although these are not as powerful pneumococcides. Similar dilutions in undiluted Dobell's solution may be used for douching or spraying the nose, or, incorporated in a dental cream, for cleansing the teeth. For washing the mouth and gargling, a solution is conveniently prepared after the following formula:

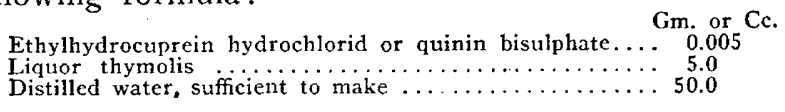

As previously stated, the systematic use of either of these mixtures may serve to destroy or inhibit the multiplication of virulent and disease producing types of pneumococci among contacts and convalescents, and thereby aid in the prophylaxis of lobar pneumonia.

\section{SUCCESSFUL TREATMENT OF ANTHRAX BY VARIOUS METHODS}

\section{G. DUDLEY, M.D.} ENDICOTT, N. Y.

I have treated anthrax successfully by three different methods. Anthrax is not always fatal. When treated properly, few, if any, patients die. The anthrax bacillus is not difficult to destroy or inactivate, but the spore resists the ordinary antiseptics and disinfectants even in highly concentrated solutions. It also resists boiling for thirty minutes and drying indefinitely. Therefore, if disinfection is done thoroughly before spore development occurs, an epidemic can be avoided; but after spore formation has taken place, the best that can be done is to destroy the bacilli as fast as they form. Spores are derived from the bacillus. Thus, while disinfection of hides and tanneries cannot affect the spore itself, it may prevent the formation of spores by breaking the cycle. In view of the fact that a suitable medium is required for spore formation, and that the spore does not form in the animal's blood, it is reasonable to assume that there are no spores in the hide of an animal dead from anthrax, and that a dry, hairy hide is not a "suitable medium" for the formation of spores. Therefore, it is possible that disinfection of hides is successful owing to the absence of spores. Obviously this should be done before spore formation takes place. One never can be certain that the disease will not break forth even sporadically years after.

Hide handlers who wear gloves are infected on the neck or face, the bacillus frequently gaining entrance by way of dead hairs, abrasions due to the razor, etc. In one case it was possible to trace a case of anthrax to the gloves worn while handling hides two weeks before, the patient in the meantime following another 
occupation. While shaving, this man broke the skin on the neck; he then put on the gloves which he had worn in the tannery, and inadvertently rubbed the abrasion on his neck. Anthrax developed quickly. The disease has also been traced to shaving brushes and fur boas.

At the site of inoculation, a small papule quickly forms. This rapidly develops into a small, dark swelling, not quite as large as a split pea, with an eschar on its summit. Soon a red halo appears, and a "blanched" zone about one-eighth inch in diameter is often present between the dark spot and the halo, resembling the area of anemia that results from the injection of cocain and epinephrin. Often there is a slight "puffiness" of the blanched area. There is no pus present, but as the lesion progresses, the eschar, which resembles burned leather and is of a hard, gummy consistency, enlarges, blebs form all around it, and these pour forth serum. Any attempt to remove the eschar at this time will prove a failure because it is firmly attached to the underlying tissues and is of such consistency that it resists cutting as well as tearing. Within a few hours the tissues become edematous to a degree out of all proportion to the size of the lesion. This edematous swelling is not hard and infiltrated, but soft. It does not pit on pressure, and offers no resistance to pressure.

There are no subjective symptoms, such as pain or itching, nor is there any fever or increase in the pulse rate while the disease is localized.

A successful excision of the lesion, removing an area about 2 or 3 inches in diameter, terminates the case. An unsuccessful attempt at excision is followed in about forty-eight hours by a slight evidence of edema at some angle of the wound, indicating that the disease is spreading in that direction. At about this time infiltration is present, but I believe that this may be due, in large measure, to the preliminary injection of phenol.

The diagnosis is based on the appearance of a papule with an ischemic zone later changing to slight puffiness, a dark, hard, resistant center and no pus, the rapid onset of the characteristic edema, and no itching, burning, pain or tenderness.

\section{TREATMENT}

I shall discuss the treatment under three heads.

1. Treatment by Excision.-As soon as the diagnosis is made, the skin is scrubbed gently with soap and rinsed thoroughly with sterile water. The skin is painted with aqueous 8 per cent. phenol (carbolic acid) solution or stronger, and rinsed with alcohol. Next the lesion is painted with collodion to avoid contamination of the incision. Eight per cent. phenol is injected into the tissues all around the lesion to wall off the infective process. From three to five syringefuls of solution (about 60 c.c.) will be sufficierit. One-fourth inch outside of this phenolized zone, five or six syringefuls of 25 per cent. alcohol are injected. These injections are usually made within $11 / 2$ inches of the center of the lesion. The line of incision is painted with 8 per cent. phenol, and an area from $2 \frac{1}{2}$ to $3 \frac{1}{2}$ inches in diameter is excised. After the excision, the base and edges of the wound are painted with pure phenol (95 per cent.) and immediately neutralized with absolute alcohol. The surface is cleansed with alcohol, and a wet dressing applied. I have used boric acid, 20 per cent. alcohol, and hypertonic saline solution.
Ordinarily this terminates the case. If, however, in forty-eight hours, slight edema appears at some angle of the wound, and in twelve hours there is reason to believe that there is still some infection present, further steps are necessary.

\section{Treatment When Excision Fails.-Injecting} three or four syringefuls of 8 per cent. phenol into the edematous zone may effect a cure. If not, the tissues are incised freely, the course of the edema being followed, and gauze drains are put in. Phenol is injected into the tissues, due regard being paid to its toxicity, and an ice bag is applied. I am indebted to Dr. John Mansuay of Ralston, Pa., for this followup method. I have never known it to fail. I always use the ice bag, although some men claim to have good results from heat.

3. Antianthrax Serum.-At this time, serum should. be used. I use the serum furnished by the U. S. Bureau of Animal Industry, Washington, D. C. The first dose is 35 c.c. injected intravenously, followed within from eight to sixteen hours by a second dose, given intramuscularly or intravenously. This dose is repeated if necessary. The only cases of chills or fever that I have ever seen in connection with anthrax occurred following this injection. In one case the temperature was as high as $106 \mathrm{~F}$., and the pulse was. 160 , but I believe that the serum had a very beneficial effect. The patient recovered.

In case the lesion is near the larynx, an attempt should be made to excise the major portion from the side toward the larynx, as patients have been known to be choked to death by the edema.

In conjunction with this treatment, I give salts in full doses, strychnin, one-thirtieth grain, every four or five hours. The patient should have plenty of fresh air, and should keep quiet. A tracheotomy tube should be kept handy in case the edema reaches the larynx.

\section{REPORT OF CASES}

CASE 1.-M. P., man, aged 47, American, tannery worker, did not work Thursday, May 24, but worked Friday and Saturday. He shaved Sunday, and at night noticed a small pimple on the nose. He reported at the office, Monday, at 10 a. m. Bacteriologic examination was positive for anthrax. The lesion was typical of anthrax, with a red streak running out under the eye, a sign of a rapidly spreading infection. I have seen two such cases. The streak resembles a burst vein in the leg or dilated capillaries often seen on the nose, Because of its nearness to the eye, it was thought impossible to excise the infected area successfully. Therefore, I injected 8 per cent. phenol into the surrounding tissues, and I made five incisions, inserted a drain in each, and applied the ice bag continuously. Thirty c.c. of antianthrax serum were given intravenously. Fourteen hours later the right eye was closed, owing to the disease and the phenol. The temperature was $101.4 \mathrm{~F}$

May 29 , at 11 a. m., the temperature was $101 \mathrm{~F}$. The swelling extended back to the ear and down on a level with the mouth. Serum was given at $11: 30$ a. $\mathrm{m}$. At midnight there was no marked change. The glands at the angle of the jaw were enlarged and tender; there was slight edema over the sternum. The temperature was $104.6 \mathrm{~F}$, the pulse, 100 .

May 30, there was slight edema of the tissue over the sternum. The glands at the angle of the jaw were diminished in size and less tender. Blebs had formed over the eye. The temperature fell during the day from 104 to $98 \mathrm{~F}$.

May 31, there was no change. Serum was given at 8 p. m. The temperature was $101.8 \mathrm{~F}$.

June 1, the swelling had nearly left the forehead. The temperature was $99 \mathrm{~F}$. The edema over the sternum was the same; there was pitting on pressure. The neck was not swollen markedly; the lips were less swollen; the patient 
could almost open the eye. The pustule was black; the blebs had burst, and there was a great discharge of serum in which were found numerous bacilli.

A third injection of serum, 20 c.c., was given.

Although the temperature rose to 104 , the pulse rate did not exceed 100. No chills or other bad symptoms were observed.

June 2, the swelling had receded, and the brawniness and redness were disappearing; the lips and the other side of the face to which the swelling had extended were normal. In two more days the man was pronounced to be out of danger.

Tuly 3, the site of the disease was healed. The patient reported at the office after having ridden 9 miles on the trolley.

CAse 2.-G. C., man, aged 40, American, April 30, noticed a small, insignificant papule over the sternocleidomastoid muscle; this case illustrates the importance of excising freely and of painting the surface with phenol. The papule was yellow, hard, and well "blanched," No eschar had formed, although the red halo had appeared. The area of edema was about 1 inch in diameter, and characteristic. Microscopically, the smear was found teeming with anthrax bacilli, more so than any $I$ had ever seen.

The routine incision was made, but we did not swab the wound with phenol, and we sutured it at once, which was an experiment made to reduce the scar.

May 1, there was no change.

May 2, the wound was puffy at the upper angle, owing, perhaps, to the phenol.

May 3, there was edema up on the cheek. The incision looked good. The temperature was $101.8 \mathrm{~F}$. At $9: 30 \mathrm{a} . \mathrm{m}$. 30 c.c. of antianthrax serum were injected, and strychnin was ordered, one-thirtieth grain, three times a day; also salts, and an ice bag was applied continuously over a wet dressing. At noon, two syringefuls of 8 per cent. phenol were injected. At $4 \mathrm{p}$. m. the swelling had not extended beyond the area injected. Multiple incisions were made, and 20 c.c. of serum were injected into the muscle.

May 4, the swelling was less on the right cheek, and it had not extended beyond the area injected with phenol. The swelling in the parotids was not noticeably changed, except that the edema had given away to some infiltration. The temperature ranged from 102 to $104 \mathrm{~F}$., and the pulse from 85 to 108 .

May 5, the temperature was $102 \mathrm{~F}$., and the pulse 112

May 6, the temperature was $99 \mathrm{~F}$, and the pulse 76 .

May 18, the patient complained of itching of the feet and legs. The lower lip was swollen, but the swelling receded in a few minutes. It was probably an angioneurotic edema. Dreams also disturbed the patient at this time.

TREATMENT OF ANTHRAX BY NORMAL BOVINE SERUM AND BY STEAM

According to V. C. Stewart of Woburn, Mass., the steam treatment consists of directing a jet of steam from a croup kettle on the pustule, the surrounding tissues being protected by asbestos. This is done several-times a day, and seems to produce good results when the disease is localized. However, Stewart states that in about 70 per cent. of his cases the bacilli were so attenuated that this is not necessary. In these cases he uses a wet dressing. If the lesion is near the eye, the eye is protected with a wet gauze underneath the asbestos. Obviously, we must resort to other treatment if the disease is generalized.

I have never employed the treatment by normal bovine serum as such. I have heated the anthrax serum made at Washington, but have no statistics that would carry weight. In estimating the value of serum treatment, we must take cognizance of the fact that some patients recover spontaneously and that some bacilli are attenuated; yet in a large series of cases treated by Kraus, it would appear that serum has marked therapeutic value.

\section{DETERMINATION OF TYPES OF PNEUMOCOCCUS IN LOBAR PNEUMONIA}

\section{a Rapid CUltural mithod *}

\author{
O. T. AVERY, M.D. \\ NEW YORK
}

In view of the fact that lobar pneumonia is prevalent in military camps in the United States, and that at present it is impossible to obtain white mice in sufficient numbers for carrying out the biologic tests necessaty in the determination of types of pneumococcus, a rapid cultural method dispensing with the use of these animals is now proposed.

Experience has demonstrated the importance of determining the immunologic type of pneumococcus concerned in the production of each individual case of lobar pneumonia. A satisfactory method for this consists in inoculating a white mouse intraperitoneally with a small amount of sputum, and in recovering the pneumococcus by cultural methods from the heart's blood after the death of the animal. The type of pneumococcus can be determined in this way in about forty-eight hours. When it became evident that specific type pneumococcus serum has distinct therapeutic value, it was necessary to devise a method by means of which the type of infecting pneumococcus could be determined in a shorter time. It has been found that when a mouse is injected with pneumonic sputum in which other organisms besides the pneumococcus are present, the pneumococci grow quickly, and the contaminating bacteria tend to disappear, or at least to be inhibited in their initial growth. Pneumococci and their products accumulate so rapidly in the peritoneal cavity of the infected mouse that after a few hours the bacterial exudate, collected by washing out the abdominal cavity, may be used in the performance of agglutination and precipitin tests by means of which the specific type of pneumococcus can be determined, often within from six to eight hours. Both of these methods are satisfactory, but both require the use of white mice.

It has long been known that the addition of carbohydrate, such as glucose, and of blood proteins to mediums suitable for cultivation of the pneumococcus greatly accelerates the initial growth of this organism. It is also known that bile causes solution of the pneumococcus and possesses no lytic action for other bacteria. Use has, therefore, been made of these fact: in devising a method for the rapid cultivation and type determination of the pneumococcus directly from the sputum of patients suffering from lobar pneumonia. These principles are employed in the following manner:

\section{METHOD}

A selected kernel of sputum, about the size of a bean, is washed, emulsified in broth, and inoculated directly into a centrifuge tube containing about 4 c.c. of special medium-glucose-blood-broth. After incubation in the water bath at $37 \mathrm{C}$. for five hours, a blood agar plate is streaked with a loopful of culture fluid for the isolation of the pneumococcus in pure culture and the subsequent confirmation of type. The red blood cells are then removed from the culture medium by slow centrifugation. Three c.c. of the

* From the Hospital of the Rockefeller Institute for Medical Research. 